\title{
Power Analysis Attack on Hardware Implementation of MAC-Keccak on FPGAs
}

\author{
Pei Luo ${ }^{1}$, Yunsi Fei ${ }^{1}$, Xin Fang ${ }^{1}$, A. Adam Ding ${ }^{2}$, and Miriam Leeser ${ }^{1}$ and David R. Kaeli ${ }^{1}$ \\ 1 Department of Electrical and Computer Engineering, Northeastern University, Boston, MA 02115 \\ silenceluo@coe.neu.edu, yfei@ece.neu.edu, fang.xi@husky.neu.edu, mel@ece.neu.edu, \\ kaeli@ece.neu.edu \\ 2 Department of Mathematics, Northeastern University, Boston, MA 02115 \\ A.ding@neu.edu
}

\begin{abstract}
Keccak is the hash function selected by NIST as the new SHA-3 standard. Keccak is built on Sponge construction and it provides a new MAC function called MAC-Keccak. These new algorithms have raised questions with regards to side-channel leakage and analysis attacks of MAC-Keccak. So far there exists prior work on attacks of software implementations of MACKeccak, but there has been no comprehensive side-channel vulnerability assessment of its hardware implementation. In this paper we describe an attack on the $\theta$ step of the first round of MACKeccak implemented on an FPGA. We construct several different side-channel leakage models and implement attacks based on them. Our work shows that an unmasked hardware implementation of SHA-3 is vulnerable to power-based side-channel attacks.
\end{abstract}

\section{1 introduction}

Keccak was selected as the winner of the NIST hash function competition in 2012 to become the SHA-3 standard [1. Keccak uses the Sponge construction 2 in which message blocks are XORed into the state bits and then invertibly permuted [3]. Sponge construction is completely different from the previous hash standards and thus opens up new questions and challenges in side-channel analysis (SCA).

A message authentication code (MAC) is a short piece of information generated by hash functions to provide integrity and authenticity assurances on the message. MAC is important in crypto systems because it facilitates integrity checking and proof of origin. Sponge construction allows Keccak to securely create a MAC by hashing the concatenation of the key and the message $(P=K|| M)$ in a cryptographic mode, called MAC-Keccak [213/4. This MAC construction is different from previous MAC methods and has attracted much attention.

Keccak has been implemented in software or a library for various general-purpose processors, embedded processors, and DSPs. In addition, there have also been hardware implementations of Keccak, which can accelerate hash computations in security-sensitive applications. As an example, a virtual network server typically uses a hash function both to verify the identity of its clients and the integrity of the messages sent by the clients. Hardware implementations of hash functions are preferred for their high throughput. High-speed integrated circuits (ICs) and FPGAs-based hash modules are widely used in cryptographic systems, Trusted Platform Modules (TPM), and crypto processors. However, the side-channel security resiliency of hardware implementations of MAC-Keccak has not been evaluated quantitatively.

In [5], six SHA-3 candidates (including Keccak) were analyzed for side-channel leakage for the first time. In the paper, the authors analyzed the feasibility of attacking MAC-Keccak at the $\theta$ step in the first round and proposed the basic steps for the attack. They presented a general side-channel attack feasibility analysis for all six candidates, though no detailed leakage models or attack methods are given for Keccak.

In [6] and [7], the side-channel vulnerability of MAC-Keccak was analyzed and it was found that side-channel resistance of MAC-Keccak depends on the length of the key used. The optimal key-length was also analyzed. The work demonstrates a practical side-channel analysis attack against MAC-Keccak implemented on a 32-bit Microblaze processor. They focused on a software implementation, but did not describe how to carry out attacks on hardware implementations. 
In additions to the bare algorithm of MAC of Keccak, the designers of Keccak also suggested methods to protect Keccak implementations from power analysis attacks 89. Random masking is an effective countermeasure. They showed that two shares are required in software implementations and three shares are required in hardware implementations.

Compared to the software implementations of MAC-Keccak [6], we anticipate much lower sidechannel leakage of hardware implementations of MAC-Keccak with their highly parallel implementations (versus the temporally spread-out software implementations). Thus, side-channel attacks on a hardware implementation of MAC-Keccak would be much more challenging to implement. Up until now, there has not been much prior work along this direction - side-channel analysis of hardware MAC-Keccak remains an open problem. Without a good understanding of the sources and amount of side-channel leakage of MAC-Keccak, any protection against side-channel attacks cannot be evaluated accurately either. To address these issues and facilitate secure MAC-Keccak design and implementation, in this paper we propose several side-channel leakage models of hardware MAC-Keccak and launch practical power analysis attacks on the implementation. To the best of our knowledge, this is the first work which develops concrete side-channel leakage models, power analysis attack methods and attack results on a hardware MAC-Keccak implementation. In light of the popular usage of Keccak as the new hash standard, our work should make a significant contribution for side-channel security analysis of SHA-3. We further discuss the factors that affect side-channel leakage of MAC-Keccak and suggest countermeasures to improve its system security.

The rest of the paper is organized as follows. In Section 2, we introduce the MAC-Keccak algorithm, the several power leakage models we adopt for attacking it, and correlation analysis results that verify the proposed leakage models. In Section 3, we implement correlation power analysis (CPA) based on the models we introduce in Section 2 to break MAC-Keccak. In Section 4, we discuss the effect of Keccak keylength on SCA and suggest countermeasures against power analysis attacks. In Section 5 , we conclude the paper.

\section{Keccak and the side-channel leakage models}

\subsection{Keccak Hash Function and MAC-Keccak}

Keccak is a hash function family based on the Sponge construction, as shown in Fig. 1 324. Keccak has two phases: 1) absorbing and 2) squeezing. In the absorbing phase, the message is broken into blocks (each block size is $r$ bits, where $r$ is the bit rate), which are absorbed iteratively by the permutation function $f$. Each $f$ function works on a state at a fixed length $b=r+c$, where the bit rate $r$ determines the implementation speed, while the capacity $c$ determines the security length. In the squeezing phase, outputs are squeezed also by $f$ functions and the length of the output is configurable (a multiple of $r$ bits).

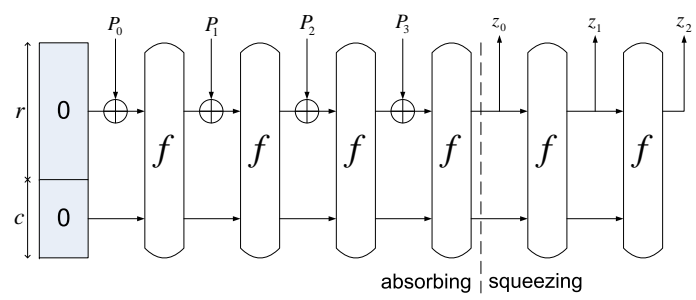

Fig. 1. The Sponge construction

When using hash functions in MAC, there exists the commonly used HMAC construction which accepts a message $M$ and a key $K$, and generates the corresponding digest [7] :

$$
H M A C(M, K)=H((K \oplus \text { opad }) \| H((K \oplus \text { ipad }) \| M)) \text {. }
$$


For Keccak, another MAC construction, MAC-Keccak [234] is recommended by the Keccak designers as:

$$
M A C(M, K)=H(K \| M) .
$$

The default Keccak mode is Keccak-1600, with $r=1024$ and $c=576$. All of the 1600-bit states are organized in a 3-D array, as shown in Fig. 2. Each bit is addressed with three coordinates, written as $S(x, y, z), x, y \in\{0,1, \ldots, 4\}, z \in\{0,1, \ldots, 63\} .2-\mathrm{D}$ entities, plane and sheet, and 1-D entities, lane and column, are also defined in Keccak and shown in Fig. 2. A plane $P_{Y}$ contains all state bits $S(x, y, z)$ for which $y=Y$; similarly, a slice $S L_{Z}=\{S(x, y, z), z=Z\}$; a sheet $S H_{X}=\{S(x, y, z), x=X\}$. A lane $L_{X, Y}$ contains all state bits $S(x, y, z)$ for which $x=X$ and $y=Y$; similarly, a column $C_{X, Z}=$ $\{S(x, y, z), x=X, z=Z\}$; a row $\operatorname{Row}_{Y, Z}=\{S(x, y, z), y=Y, z=Z\}$.

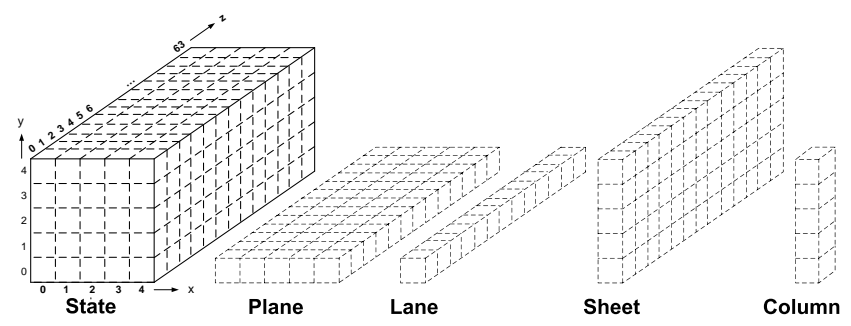

Fig. 2. Terminology used in Keccak

The $f$ permutation functions of Keccak-1600 consist of 24 rounds of operations, where each round has five sequential steps:

$$
R_{i+1}=\iota \circ \chi \circ \pi \circ \rho \circ \theta\left(R_{i}\right), i \in\{0,1, \cdots, 23\}
$$

in which $R_{0}$ is the initial input.

Details of each step are described below:

$-\theta$ is a linear operation which involves 11 input bits and outputs a single bit. Each output state bit is the XOR between itself and two intermediate bits produced by its two neighbor columns. The operation is given as follows:

$$
\begin{aligned}
S^{\prime}(x, y, z)=S(x, y, z) & \oplus\left(\oplus_{i=0}^{4} S(x-1, i, z)\right) \\
& \oplus\left(\oplus_{i=0}^{4} S(x+1, i, z-1)\right) .
\end{aligned}
$$

The two intermediate bits are the parity of the two columns, $\oplus_{i=0}^{4} S(x-1, i, z)$ and $\oplus_{i=0}^{4} S(x+1, i, z-1)$, respectively.

$-\rho$ and $\pi$ are permutations over the bits of the state.

$-\chi$ is a non-linear step that contains mixed binary operations. Every bit of the output state is the result of an XOR between itself and the AND result of one neighboring bit and the NOT of another neighboring bit along the $\mathrm{x}$-axis:

$$
S^{\prime}(x, y, z)=S(x, y, z) \oplus(\overline{S(x+1, y, z)} \cdot S(x+2, y, z)) .
$$

$-\iota$ is a binary XOR with a round constant.

$\theta$ is the first step in each round and thus the $\theta$ operation in the first round of MAC-Keccak involves the secret key and the known message $(K \| M)$ directly. Intermediate variables that are dependent on $K$ may result in side-channel leakage. Therefore we focus on $\theta$ in the first round for power analysis. Details of $\rho, \pi, \chi$ and $\iota$ can be found in [1]2]3/4]. 


\subsection{The $\theta$ Operation}

For the standard VHDL implementation Version 3.1 provided online by keccak.noekeon.org [10, each round takes one clock cycle and the five steps $(\theta, \rho, \pi, \chi$ and $\iota)$ are all implemented in combinational circuits. In this paper, we attack the $\theta$ step of the first round.

As described in [6], $\theta$ operation is computed over two successive steps. In the first step, $\theta_{1}$ calculates the parity of each column and compresses a 1600 -bit state into a 320 -bit plane called the $\theta_{\text {plane }}$ :

$$
\begin{gathered}
\theta_{\text {plane }}(x, z)=\oplus_{y=0}^{4} S(x, y, z) \\
x \in\{0,1, \ldots, 4\}, z \in\{0,1, \ldots, 63\} .
\end{gathered}
$$

$\theta_{1}$ can be viewed as 320 independent operations, each of which XORs the five bits in a column to generate one bit of parity.

In the second step, $\theta_{2}$ computes the XOR between every bit of the state and two neighboring parity bits of the $\theta_{\text {plane }}$, and outputs $\theta_{\text {out }}$.

$$
\begin{gathered}
\theta_{\text {out }}(x, y, z)=S(x, y, z) \oplus \theta_{\text {plane }}(x-1, z) \\
\oplus \theta_{\text {plane }}(x+1, z-1) \\
x, y \in\{0,1, \ldots, 4\}, z \in\{0,1, \ldots, 63\} .
\end{gathered}
$$

\subsection{Side-channel Leakage Models}

In the hardware implementation of MAC-Keccak, all the five steps in each round are executed in one clock cycle, and the intermediate variables used for attacks on the software implementations may not be correlated with the power traces. Thus we cannot use the leakage models for software implementations 617] directly for hardware systems. For the standard hardware implementation of MAC-Keccak [10, we will describe the four side-channel leakage models in this section.

The structure of the standard Keccak hardware implementation [10] is shown in Fig. 3, where one state register holds both the input and output of a round operation. In the round operations, the first round loads the message $P$ in using a multiplexer, and other rounds take the state register output as input to the following five-step operation. Note here that after performing padding, $M$ includes both the message bits and appended padding bits. For the simplified setting in this paper, we set the length of $K \| M$ as 1024 bits, thus there is only one message block. We still use $P$ to denote the input to the first round for convenience. $\theta$ can be split further into $\theta_{1}$ and $\theta_{2}$, and the other four steps are considered together in a combinational module. We denote the latency of the $\theta_{1}$ operation as $t_{1}$ and the latency of the $\theta_{2}$ operation as $t_{2}$.

For the standard hardware implementation, we describe the operations and discuss important time points in the first cycle:

- The state register reg is cleared before each MAC-Keccak operation, thus $O\left(\theta_{1}\right)$ and $O\left(\theta_{2}\right)$ are initialized at 0 . At time 0 , the message and key after padding $(P)$ is loaded into the output of MUX (we ignore the delay of the MUX here).

- At $t_{2}, O\left(\theta_{2}\right)$ changes from 0 to $P$, because its input from $O(M U X)$ already changed to $P$ while the other input ( $\theta_{1}$ 's output) remained 0 .

- At $t_{1}, O\left(\theta_{1}\right)$ changes from 0 to $\theta_{\text {plane }}$.

- At $t_{1}+t_{2}, O\left(\theta_{2}\right)$ changes from $P$ to $\theta_{\text {out }}$ as a response to the input $O\left(\theta_{1}\right)$ 's changing from 0 to $\theta_{\text {plane }}$.

In general, for hardware implementations (either FPGA or ASIC), the power model of an intermediate variable is the Hamming Distance (HD) model, i.e., the power consumption of this intermediate state and the operation driving the state is linearly dependent on the HD between the value before the operation and the value after the operation. We then have the following 2 side-channel leakage models:

1. Model I: At $t_{1}$, the intermediate variable $O\left(\theta_{1}\right)$ changes from 0 to $\theta_{\text {plane }}$, and thus the HD is $H W\left(\theta_{\text {plane }}\right)$. There should exist a time point on the power trace that has a good correlation between its power value and $H W\left(\theta_{\text {plane }}\right)$. 


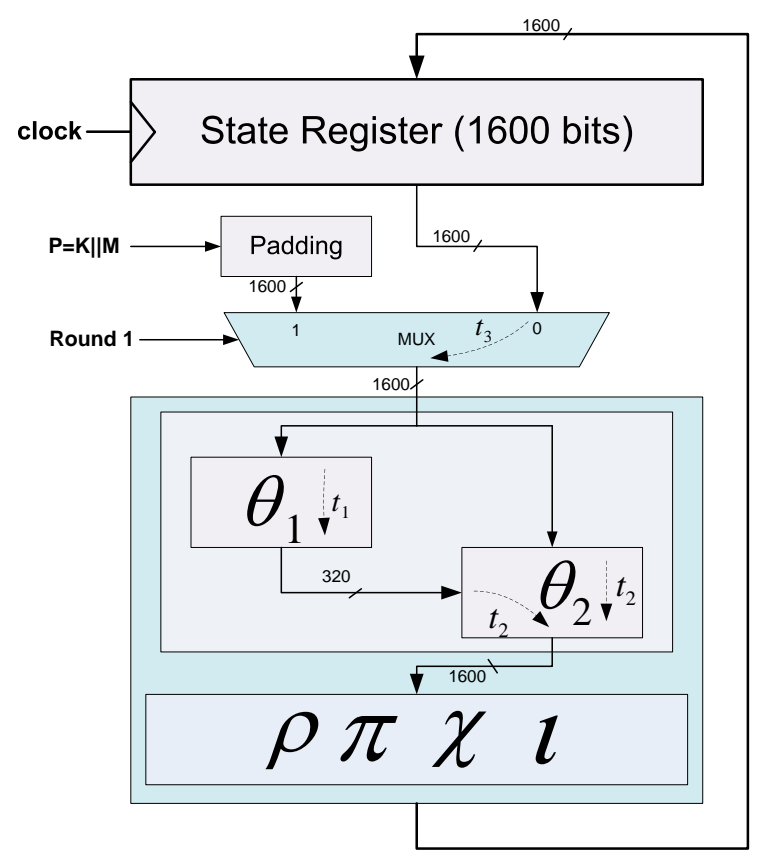

Fig. 3. Structure of Keccak hardware implementation

2. Model II: At $t_{1}+t_{2}$, the intermediate variable $O\left(\theta_{2}\right)$ changes from $P$ to $\theta_{\text {out }}$, and the HD is $H D\left(P, \theta_{\text {out }}\right)$. Similarly, there should exist a time point on the power trace that has a good correlation between its power value and $H D\left(P, \theta_{\text {out }}\right)$.

To verify the above side-channel leakage models, we implemented the official VHDL description of Keccak 1011] on an Sasebo-GII 12 board which contains a Xilinx Virtex-5 FPGA. We use the Xilinx ISE 14.6 for implementation with all of the default settings for synthesis, mapping and routing. From the post-mapping results, we find that there are 320 look-up-tables (LUTs) for the $\theta_{1}$ module, and just 320 LUTs for the top plane in the $\theta_{2}$ module instead of 1,600 LUTs for all the $\theta_{\text {out }}$ bits. This is because the synthesis tool optimized the implementation and combines other parts of $\theta$ with following operations $(\rho, \pi, \chi$ and $\iota)$. Thus we anticipate that the leakage for Model II will be weaker than expected.

Then we collect power traces from our MAC-Keccak implementation running at $12 \mathrm{MHz}$ using an Agilent MSOX4104A oscilloscop. We use 700,000 traces to find correlations based on Models I and II. We show one power trace and Pearson correlation 1314 results in Fig. 4. Fig. 4(a) shows one example power trace of MAC-Keccak, where the first trough corresponds to the loading of message $M$ into the padding module and then $P$ to the $\theta$ module. From Fig. $4(\mathrm{~b})(\mathrm{c})$, we can see that the side-channel power leakage for Model I and Model II are both noticeable (with the highest correlation reaching -0.09 and -0.03 respectively) in the first cycle (shown by two dashed lines). Meanwhile, we notice an even stronger correlation between the $H D\left(P, \theta_{\text {out }}\right)$ and the power at the beginning of the second round (with the highest correlation reaching -0.2 , as shown in Fig. $4(\mathrm{c}))$. This point may be the moment the contents of the state register change from 0 to $R_{1}$.

We anticipate some leakage signals in the second round as well. Similar to the first cycle, we identifies the operations and associated time points in the second cycle.

- At the beginning of the clock cycle, the state register stores the first round result, and the contents of the state register change from 0 to $R_{1}$.

- At $t_{3}$ (the latency of multiplexer), the multiplexer selects the result $R_{1}$ and passes it on to the output, therefore $O(M U X)$ changes from $P$ (for the first round operations) to $R_{1}$. 

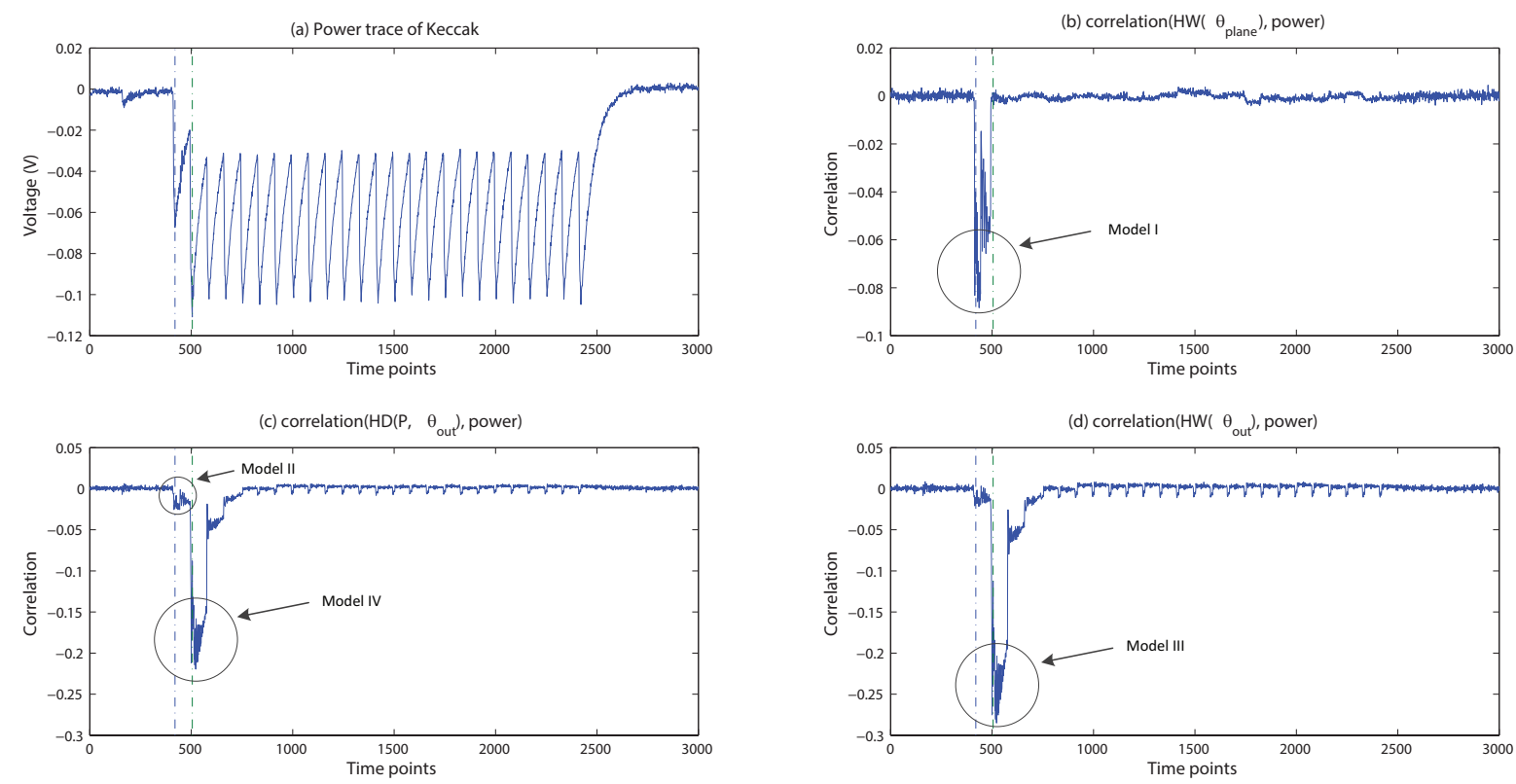

Fig. 4. Power trace and the correlations

However, $R_{1}$ is the result of the 5 -step operation which includes the $\theta$ step and others $(\rho, \pi, \chi, \iota)$. The key-dependence of $R_{1}$ should be random, according to the hash function design principle. We try to seek leakage signals related to $\theta_{\text {out }}$ (rather than $R_{1}$ ) that contain the key information during the second round.

Surprisingly, Fig. 4 (c)(d) show that there are actually time points on the power traces strongly correlated with $H W\left(\theta_{\text {out }}\right)$ and $H D\left(P, \theta_{\text {out }}\right)$. We hypothesize that due to the inherent algorithmic correlation between $\theta_{\text {out }}, R_{1}$ and $P$, we have two more power leakage models for the second cycle:

1. Model III: The state register output changes from 0 to $R_{1}$. There exists a time point in the second clock cycle that is correlated with $H W\left(\theta_{\text {out }}\right)$ (actually $H W\left(R_{1}\right)$ ).

2. Model IV: The output of multiplexer changes from $P$ to $R_{1}$. There exists a time point in the second clock cycle that is correlated with $H D\left(P, \theta_{\text {out }}\right)$ (actually $\left.H W\left(R_{1}\right)\right)$.

To verify the previous hypothesis about correlations, we randomly generated 200,000 messages and ran Keccak on them. The correlation results are as follows:

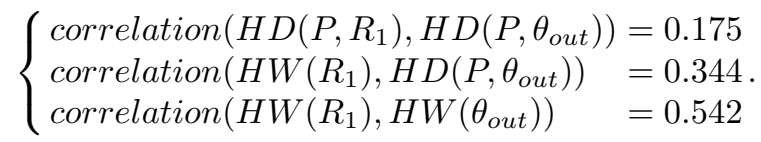

We find that the correlation between $H W\left(R_{1}\right)$ and $H W\left(\theta_{\text {out }}\right)$ is higher, which also explains why Model III has a stronger leakage (with correlation reaching -0.29) than Model IV (with correlation reaching -0.22). Models II, III and IV all involve $\theta_{\text {out }}$ and extract the same key information. Therefore, we choose to use Model III, which has the highest correlation (attack effectiveness) for our attacks.

Here even if the state register is initialized to the first round input $P$, the leakages of $\theta_{\text {out }}$ still exist because of the algorithm properties. Our attack is not restricted to the case of state register initialized to 0 only. 


\section{Side-channel attacks on MAC-Keccak}

In this section, we show how to launch CPA on MAC-Keccak to recover the key bits. Keccak allows the use of a variable-length key. We start with a key length of 320 bits (i.e., the key fills the bottom plane in the input state, and the message length is $1024-320=704$ bits). The Sponge function (shown in Fig. 1) contains only one $f$ function. We will discuss the effect of key length on attacks in Section 4 .

\subsection{CPA based on Model III}

Next, we show how to extract the relationship between every two lanes of the key plane, based on power Model III.

For Model III, $P=K \| M$ where the unknown part is the key $K$ (320 bits), and the known part is the message $M$ (704 bits). According to (7), the Hamming weight for a single bit in model III is:

$$
\begin{aligned}
& H W\left(\theta_{\text {out }}(x, y, z)\right)=H W\left(S(x, y, z) \oplus \theta_{\text {plane }}(x-1, z)\right. \\
& \left.\oplus \theta_{\text {plane }}(x+1, z-1)\right)=H W(S(x, y, z) \oplus \\
& \left.\left(\oplus_{y=0}^{4} S(x-1, y, z)\right) \oplus\left(\oplus_{y=0}^{4} S(x+1, y, z-1)\right)\right) .
\end{aligned}
$$

Equation (9) shows that the operation is on two neighboring columns of the bit, which include 8 bits of the known $M$ and 2 bits of the unknown $K$. This side-channel power leakage will give information related to the XOR of these 2 unknown key bits, $S(x-1,0, z) \oplus S(x+1,0, z-1)$, to the attacker.

To increase the leakage signal strength of CPA, instead of using 1 bit in a column for the select function, we use 4 bits $(y=1,2,3,4)$ instead. To make a balance between the leakage signal and the attack complexity, each time we can attack multiple bits $(l, 0<l \leq 64)$ in each key lane. The larger the $l$, the stronger the leakage signal, but also the higher complexity due to enumeration of the key bits. The multi-bit Hamming weight model (III) becomes:

$$
\begin{array}{r}
\sum_{z=i}^{i+l-1} \Sigma_{y=1}^{4} H W\left(\theta_{\text {out }}(x, y, z)\right) \\
i \in\{0,1, \ldots, 63\}, l \in\{1,2, \ldots, 64\}
\end{array}
$$

and we can recover $S(x-1,0, Z) \oplus S(x+1,0, Z-1), x \in\{0,1, \cdots, 4\}, Z=[i: i+l-1]$.

We choose $l=8$ along the key lane, and run CPA based on Model III to recover the relationship between the two key bytes. One CPA attack instance and the empirical success rate results are shown in Fig. 5 .

Fig. 5 (a) shows the correlation analysis result for key guesses based on Model III using 100, 000 traces. It shows that the correct key candidate has the highest correlation, reaching 0.055 . The correlation trace for MAC-Keccak is very different from those for previous crypto algorithms such as AES or DES. This is because CPA performed on block ciphers such as AES attacks SBox, which is a non-linear operation and a small difference on the key guesses results in a big difference in the select function value. For MAC-Keccak, $\theta$ is a linear operation and one bit key change has a limited affect on the Hamming weight of the output value. Fig. 5(b) gives the CPA success rates (SRs) based on Model III, which include four modes: the correct key is ranked top one according to the correlation result, ranked in the top 2, top 4 and top 8, respectively. Fig. 5(b) shows that the success rate increases when we use a less accurate ranking. The success rates all approach to $100 \%$, and require 10,000 traces for ranking in top $8,20,000$ traces for ranking in top 2, and about 30,000 traces for top 1.

Using this method, we can recover $K(x-1, Z) \oplus K(x+1, Z-1)$, in which $K(x, Z)(x \in\{0,1, . ., 4\}, Z=$ $[0: 63])$ is one lane of the key plane. Thus, if we know one key lane, we can recover all 320 key bits in the key plane. This attack model can efficiently decrease the complexity of key guessing from $2^{320}$ to $2^{64}$ for Keccak-1600. Although $2^{64}$ is a complexity attackable by enumeration with today's computational power, in the next part, we show how to recover one lane of key bits in CPA to avoid key enumeration with the complexity of $2^{64}$, and thus totally recover the key bits. 

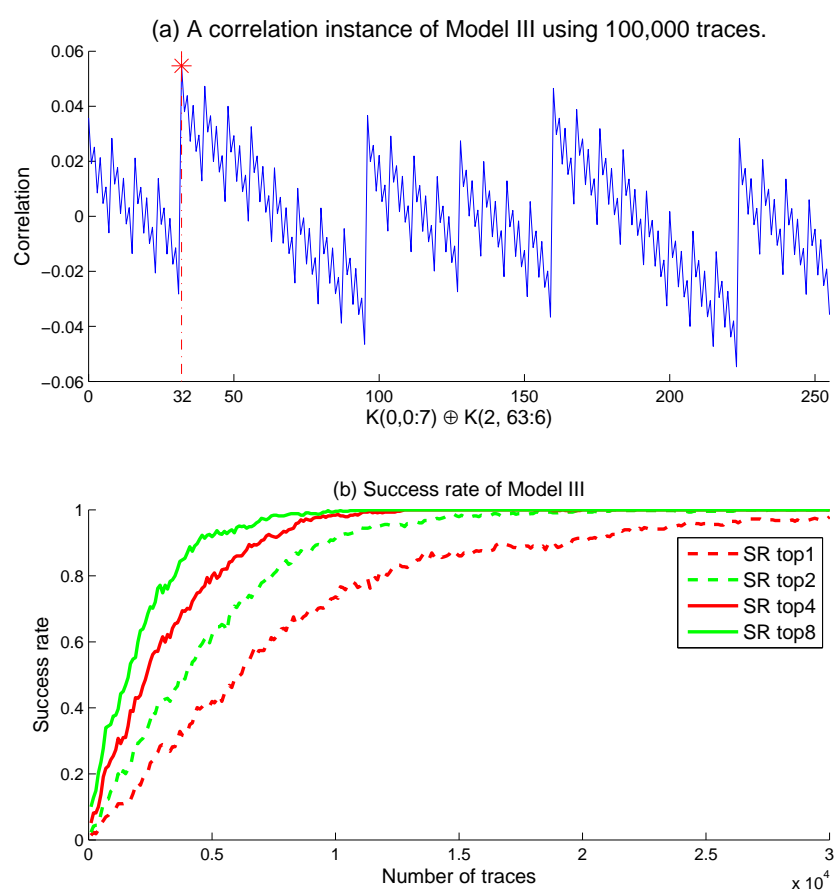

Fig. 5. Success rate curves based on model III

\subsection{Recover key bits of one lane based on Model I}

In leakage Model I, the correlation is between power and $H W\left(\theta_{\text {plane }}\right)$, and $\theta_{1}$ does not involve operations between two key lanes. According to (6), each bit of the $\theta_{\text {plane }}$ is decided by 4 bits of $M$ and 1 bit of $K$ in one column. Thus, we can use this model to recover the key bits in one key lane directly.

For a single key bit, the SNR may be very low for (6), and more than one key bit should be attacked concurrently to increase the signal-to-noise ratio (SNR). To strike a balance between SNR and complexity, we attack 8 bits for each key lane at once. For the first 8 bits in lane $x$, the Hamming weight is:

$$
H W\left(\theta_{\text {plane }}(x, 0: 7)\right)=\Sigma_{z=0}^{7}\left(\oplus_{y=0}^{4} S(x, y, z)\right) .
$$

The CPA model is:

$$
\operatorname{correlation}\left(\Sigma_{z=0}^{7}\left(\oplus_{y=0}^{4} S(x, y, z)\right), \text { power }\right) \text {. }
$$

The key byte guess with the highest correlation is the right key byte. The success rates of CPA, based on the above attack model, are shown in Fig. 6 .

From Fig. 6 we can see that when using about 500, 000 traces, we can recover one byte of one key lane with SR around $90 \%$ while there are 8 bytes in each lane. The success rates are much lower for Model I than for Model III due to the weaker correlation. Using this method, we can recover all 64 bits in one lane. Combined with the SCA method in Section 3.1. we can recover the other key lanes to get the whole key plane.

In summary, we use more than one side-channel leakage model (two complementary models) to recover all the key bits for a simplified MAC-Keccak (1600) setting. In the above scenarios, we attack 8 bits at one time and the results showed that we can recover the relationship between two lanes with only 30,000 traces and recover one key byte with SR of around 90\% using 500, 000 traces. 


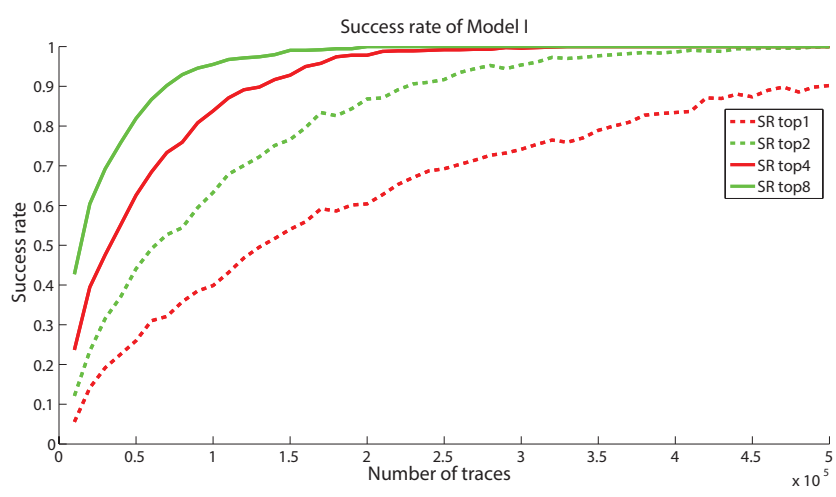

Fig. 6. Success rate based on model I

\section{Further discussion about the key-length and countermeasures}

In this section, we extend the results found in the previous experiments and analyze SCA with different key lengths. Then we discuss countermeasures against this kind of power analysis attacks.

\subsection{Different Parameters for MAC-Keccak}

Note that the official hardware implementation [10 has different parameters (rate and capacity) from FIPS 202 [1], which was only published recently in April 2014. These parameter differences will not affect the SCA of MAC-Keccak because the power leakage models and attack methods remain the same.

\subsection{Key Length}

In our FPGA implementation of MAC-Keccak, we assumed that the key length is 320 bits, which is just the size of one plane such that there will be no operation between key bits in the same column. Previous work [6] discussed the effect of key length on SCA. Here, we will summarize the effect of key length on SCA under our leakage and attack models.

- key-length $\leq$ plane-size $\times 0.8$ : In this situation, the key bits take no more than 4 lanes in the key plane. For Keccak-1600, it means key-length $<256$, and there is interaction between key lanes and known message lanes in the bottom plane. One leakage model (Model III or IV) is enough to recover all the key bits.

- plane-size $\times 0.8<$ key-length $\leq$ plane-size: In this situation, only one lane is mixed with key bits and message bits in the first plane. The leakage models (I and III) have to be combined to recover the key bits. We used 320 bits as the key length to describe our attacks in previous sections.

- key-length > plane-size: In this situation, the key bits occupy more than the first plane. Models I, II, III, and IV will involve more than two key bits for a single bit $\theta$ operation. Thus the attack complexity increases rapidly as described in [7]. We will investigate concrete attacks in this scenario in future work.

\subsection{Extend the Attack Methods}

In this paper, we attack the $\theta$ step because it is the first step of the round operations and it involves the key bits directly. Its linearity allows us to break up the keys into blocks of any chosen size, to balance the effect of SNR and computational complexity. Attacks can also be launched on other steps, such as the non-linear $\chi$ step, as described in [6]. For nonlinear operations, how to choose the select function 
and intermediate variables that carry meaningful key information would be a challenge. In addition, the leakage models and correlations would all be different from the $\theta$ step, as shown in Fig. 5 .

In this work, we apply the usual CPA by choosing the key candidates with the highest correlations as the correct key, shown in Fig. 5. The linearity of $\theta$ leads to a structure in the correlation patterns in Fig. 5(a). We can use this pattern to improve the CPA attack by selecting multiple crest points in the correlation trace and use the peak points to speculate the correct key. This is because all the crest points should have very small difference from the true key (e.g., 1 bit difference). These crest points can give information about the right key to avoid key enumeration when the number of traces are limited. We will investigate this in future work.

\subsection{Countermeasures}

General protection schemes for hardware cryptographic systems include power-balanced circuit [1516, masking [9], and randomization [17/18. In this section, we discuss countermeasures for Keccak, specifically based on the previously described leakage and attack models. General protection methods, such as power balanced circuits, are omitted here.

As described in Section 4.2. MAC-Keccak with key-length $\leq$ plane-size is insecure and the attack difficulty increases rapidly with the key length. Thus the first and easiest countermeasure against SCA is to increase the key-length above a plane size.

The second countermeasure is using masking to increase the attack complexity, as described in [8 8 [19]. This makes the power consumption not only depend on the key bits and plaintext, but also the random numbers generated inside the FPGA and IC devices. Results in [9] and [13] show that the number of traces required for SCA in a masked system is much larger than for a unprotected implementation. Thus masking should be implemented on both the linear and nonlinear parts to hide the secret information from attackers.

Another concern for the hardware implementation is the initial state of the state register. The leakage models proposed in Section 2 are based on the official hardware implementation [10] which has the state register initialized to 0 . The state register changes from 0 to $R 1$ still leaks key information as shown in Section 2. To avoid such leakage, the state register can be initialized to a random number which is unknown to the attackers, and thus the attack difficulty will be much higher. All the random number generations will incur hardware and execution time overhead though.

\section{5 conclusion}

In this paper, we explore multiple side-channel leakage models of MAC-Keccak and implement CPA based on these models. Results show that MAC-Keccak has strong side-channel leakages and these leakages can be used by attackers to extract the secret key. We discuss factors which affect side-channel leakage and countermeasures against SCA. For future work, we will investigate additional and more efficient attacks on general MAC-Keccak implementations (with different key lengths, bit rates and capacities). We will also design secure MAC-Keccak with effective countermeasures, and assess their security thoroughly.

All power traces used in this paper are publicly available at http://tescase.coe.neu.edu.

\section{Acknowledgment}

This work is supported in part by NSF under grants SaTC-1314655 and MRI-1337854.

\section{References}

1. N. F. Pub, "DRAFT FIPS PUB 202: SHA-3 standard: Permutation-based hash and extendable-output functions," Federal Information Processing Standards Publication, 2014.

2. G. Bertoni, J. Daemen, M. Peeters, and G. Assche, "Cryptographic sponge functions," Submission to NIST (Round 3), 2011. 
3. G. Bertoni, J. Daemen, M. Peeters, and G. Van Assche, "Keccak sponge function family main document," Submission to NIST (Round 2), 2009.

4. G. Bertoni, J. Daemen, M. Peeters, and G. Assche, "The Keccak reference," Submission to NIST (Round 3), January, 2011.

5. M. Zohner, M. Kasper, M. Stottinger, and S. A. Huss, "Side channel analysis of the SHA-3 finalists," in Design, Automation 83 Test in Europe Conference \& Exhibition (DATE), 2012, pp. 1012-1017.

6. M. M. Taha and P. Schaumont, "Differential power analysis of MAC-Keccak at any key-length." in International Workshop on Security, 2013, pp. 68-82.

7. M. Taha and P. Schaumont, "Side-channel analysis of MAC-Keccak," in IEEE Int. Symposium on HardwareOriented Security and Trust (HOST), 2013, pp. 125-130.

8. G. Bertoni, J. Daemen, M. Peeters, and G. Van Assche, "Building power analysis resistant implementations of Keccak," in Second SHA-3 candidate conference. Citeseer, 2010.

9. G. Bertoni, J. Daemen, N. Debande, T.-H. Le, M. Peeters, and G. Van Assche, "Power analysis of hardware implementations protected with secret sharing," in IEEE/ACM International Symposium on Microarchitecture Workshops (MICROW), 2012, pp. 9-16.

10. Keccak Hardware implementation in VHDL Version 3.1, 2014 (accessed May 14, 2014). [Online]. Available: http://keccak.noekeon.org/KeccakVHDL-3.1.zip

11. "SHA-3 FPGA implementation," http://satoh.cs.uec.ac.jp/SAKURA/research/SHA-3.html.

12. "Evaluation environment for side-channel attacks," http://www.risec.aist.go.jp/project/sasebo/

13. A. Ding, L. Zhang, Y. Fei, and P. Luo, "A statistical model for higher order DPA on masked devices," in Cryptographic Hardware and Embedded Systems CHES 2014, 2014, vol. 8731, pp. 147-169.

14. Y. Fei, Q. Luo, and A. A. Ding, "A statistical model for DPA with novel algorithmic confusion analysis," in Cryptographic Hardware and Embedded Systems-CHES 2012, pp. 233-250.

15. K. Tiri and I. Verbauwhede, "A logic level design methodology for a secure DPA resistant ASIC or FPGA implementation," in Proceedings of the conference on Design, automation \& test in Europe, 2004, p. 10246.

16. S. Guilley, P. Hoogvorst, Y. Mathieu, R. Pacalet, and J. Provost, "CMOS structures suitable for secured hardware," in Proceedings of the conference on Design, Automation and Test in Europe, 2004, p. 21414.

17. M.-L. Akkar and C. Giraud, "An implementation of DES and AES, secure against some attacks," in Cryptographic Hardware and Embedded Systems (CHES), 2001, pp. 309-318.

18. P. Luo, A. Y.-L. Lin, Z. Wang, and M. Karpovsky, "Hardware implementation of secure Shamirs secret sharing scheme," in IEEE International Symposium on High-Assurance Systems Engineering, 2014, pp. 193-200.

19. B. Bilgin, J. Daemen, V. Nikov, S. Nikova, V. Rijmen, and G. Van Assche, "Efficient and first-order DPA resistant implementations of Keccak," in Smart Card Research and Advanced Applications, 2014, pp. 187-199. 